\title{
On Legendrian graphs
}

\author{
DANIELLE O'DONNOL \\ Elena PAVElescu
}

\begin{abstract}
We investigate Legendrian graphs in $\left(\mathbb{R}^{3}, \xi_{\text {std }}\right)$. We extend the Thurston-Bennequin number and the rotation number to Legendrian graphs. We prove that a graph can be Legendrian realized with all its cycles Legendrian unknots with $\mathrm{tb}=-1$ and rot $=0$ if and only if it does not contain $K_{4}$ as a minor. We show that the pair (tb, rot) does not characterize a Legendrian graph up to Legendrian isotopy if the graph contains a cut edge or a cut vertex. When we restrict to planar spatial graphs, a pair (tb, rot) determines two Legendrian isotopy classes of the lollipop graph and a pair (tb, rot) determines four Legendrian isotopy classes of the handcuff graph.
\end{abstract}

57M25, 57M50; 05C10

\section{Introduction}

We begin the systematic study of Legendrian graphs in $\mathbb{R}^{3}$ with the standard contact structure. These are embedded graphs that are everywhere tangent to the contact planes. Legendrian graphs have appeared naturally in several important contexts in the study of contact manifolds. They are used in Giroux's proof of existence of open book decompositions compatible with a given contact structure [8]. Eliashberg and Fraser [5] used Legendrian graphs to prove that in a tight contact structure the unknot is determined up to Legendrian isotopy by the invariants tb and rot. Yet no study of Legendrian graphs, until now, has been undertaken. We remedy this by establishing the foundations for what we expect will be a very rich field.

Throughout this paper we consider finite graphs. A spatial graph is an embedding of a graph in $\mathbb{R}^{3}$. An abstract graph is a set of vertices together with a set of edges between them, without any specified embedding. We refer to an abstract graph as simply a graph. In Section 3, we address the question of existence of Legendrian embeddings of spatial graphs and we prove the following:

Theorem 1.1 Given any embedded graph $G$ in $\mathbb{R}^{3}$, there exists a Legendrian realization of $G$ in $\left(\mathbb{R}^{3}, \xi_{\text {std }}\right)$. 
We then extend the classical invariants, the Thurston-Bennequin number (tb) and the rotation number (rot), from Legendrian knots to Legendrian graphs.

Mohnke [11] proved that the Borromean rings and the Whitehead link cannot be represented by Legendrian links of trivial unknots, that is, unknots with tb $=-1$ and $r o t=0$. The trivial unknot is the one unknot that attains the maximal ThurstonBennequin number of its topological class. As an application of our invariants, we ask which graphs admit Legendrian embeddings with all cycles trivial unknots. In Section 4, we give a full characterization of these graphs in the form of the following:

Theorem 1.2 A graph $G$ admits a Legendrian embedding in $\left(\mathbb{R}^{3}, \xi_{\text {std }}\right)$ with all its cycles trivial unknots if and only if $G$ does not contain $K_{4}$ as a minor.

The proof of this theorem relies partly on the fact that the trivial unknot has an odd Thurston-Bennequin number, that is, $t b=-1$. We prove the reverse implication in more generality, for $L_{\text {odd }}$. This represents the set of topological knot classes with odd maximal Thurston-Bennequin number.

Theorem 1.3 Let $G$ be a graph that contains $K_{4}$ as a minor. There does not exist a Legendrian realization of $G$ such that all its cycles are knots in $L_{\text {odd }}$ realizing their maximal Thurston-Bennequin number.

It is known that certain Legendrian knots and links are determined by the invariants tb and rot: the unknot by Eliashberg and Fraser [5], the torus knots and the figure eight knot by Etnyre and Honda [6] and the links consisting of an unknot and a cable of that unknot by Ding and Geiges [3]. In Section 5, we ask what types of spatial graphs are classified up to Legendrian isotopy by the pair (tb, rot) and we prove the following theorem.

Theorem 1.4 No graph containing at least one cycle and at least one cut edge or one cut vertex is determined up to Legendrian isotopy by the pair (tb, rot).

This means that even uncomplicated graphs carry more information than the set knots represented by their cycles.

We consider the lollipop graph and the handcuff graph. In order to have a classification by the pair (tb, rot), we must first narrow to a specific topological class. We investigate planar spatial graphs; these are embedded graphs that are ambient isotopic to planar embeddings. Not to be confused with planar graphs, which refers to an abstract graph that has a planar embedding. For topological planar graphs, in the case of the handcuff graph there are exactly four Legendrian realizations for each pair (tb, rot) and in the case of the lollipop graph there are exactly two Legendrian realizations for each pair $(\mathrm{tb}, \mathrm{rot})$. 
Acknowledgements The authors would like to thank Tim Cochran for his support and interest in the project, John Etnyre for helpful conversations and the referee for carefully reading the paper and for providing constructive comments.

\section{Background}

\subsection{Spatial graphs}

A spatial graph is an embedding $f$ of a graph $G$ in $\mathbb{R}^{3}$ (or $S^{3}$ ), also called a spatial embedding or graph embedding. We remind the reader that an abstract graph, one without an embedding, will be referred to as simply a graph. We will be considering spatial graphs in $\mathbb{R}^{3}$ throughout this paper. Two spatial graphs $f(G)$ and $\bar{f}(G)$ are ambient isotopic if there exits an isotopy $h_{t}: \mathbb{R}^{3} \rightarrow \mathbb{R}^{3}$ such that $h_{0}=$ id and $h_{1}(f(G))=\bar{f}(G)$. Similar to knots, there is a set of Reidemeister moves for spatial graphs described by Kauffman [9].

Here we remind the reader of some basic graph theoretic terminology. We are considering the most general of graphs, so there can be multiedges (edges that go between the same pair of vertices) and loops (edges that connect a vertex to itself). The valence of a vertex is the number of endpoints of edges at the given vertex. Two vertices are adjacent if there is an edge between them. The complete graph on $n$ vertices, $K_{n}$, is the graph with $n$ vertices and with exactly one edge between each pair of vertices. A graph $H$ is a minor of $G$ if $H$ can be obtained from a subgraph of $G$ by a finite number of edge contractions.

Since we are considering topological questions about Legendrian graphs throughout this paper, it is important to be aware of intrinsic properties of graphs. A property is called intrinsic if every embedding of $G$ in $\mathbb{R}^{3}$ (or $S^{3}$ ) has the property. A graph $G$ is minor minimal with respect to a property if $G$ has the property but no minor of $G$ has the property. Such properties are characterized by their full set of minor minimal graphs. A classical example of this is Kuratowski's Theorem [10] where nonplanar graphs are characterized. A graph $G$ is nonplanar if there does not exist a planar embedding of $G$. Kuratowski's Theorem says, the complete graph on five vertices $K_{5}$ and the complete bipartite graph $K_{3,3}$ are the minor minimal set of graphs for the property of nonplanarity.

A spatial graph is said to contain a knot (or link) if the knot (or link) appears as a subgraph of $G$. A graph $G$ is intrinsically knotted if every embedding of $G$ in $\mathbb{R}^{3}$ (or $S^{3}$ ) contains a nontrivial knot. A link $L$ is split if there is an embedding of a 2-sphere $F$ in $\mathbb{R}^{3} \backslash L$ such that each component of $\mathbb{R}^{3} \backslash F$ contains at least one 
component of $L$. A graph $G$ is intrinsically linked if every embedding of $G$ in $\mathbb{R}^{3}$ (or $S^{3}$ ) contains a nonsplit link. The combined work of Conway and Gordon [2], Sachs [13] and Robertson, Seymour and Thomas [12] fully characterized intrinsically linked graphs. They showed that the Petersen family is the complete set of minor minimal intrinsically linked graphs. Unlike the intrinsically linked graphs, the set of intrinsically knotted graphs has not been characterized.

These three intrinsic properties: nonplanarity, being intrinsically linked, and being intrinsically knotted are distinct and form a hierarchy of complexity. It is known that all intrinsically knotted graphs are intrinsically linked [12]. It is obvious that all intrinsically linked graphs are nonplanar. So we see that

$\{$ nonplanar graphs $\} \supset\{$ intrinsically linked graphs\} $\supset$ \{intrinsically knotted graphs\}.

We return to these issues when considering Theorem 4.3 and Corollary 4.4.

\subsection{Legendrian knots}

Let $M$ be an oriented 3-manifold and $\xi$ a 2-plane field on $M$. Then $\xi$ is a contact structure on $M$ if $\xi=\operatorname{ker} \alpha$ for some 1 -form $\alpha$ on $M$ satisfying $\alpha \wedge d \alpha>0$.

On $\mathbb{R}^{3}$, the 1 -form $\alpha=d z-y d x$ defines a contact structure called the standard contact structure, $\xi_{\text {std }}$. There is a diffeomorphism of $\mathbb{R}^{3}$ taking the standard contact structure $\xi_{\text {std }}$ to the symmetric contact structure $\xi_{\text {sym }}$ given in cylindrical coordinates by $\alpha_{1}=d z+r^{2} d \theta$. In this paper, we switch between $\xi_{\text {std }}$ and $\xi_{\text {sym }}$ when convenient. Darboux's theorem says that any contact structure on a manifold $M$ is locally diffeomorphic to $\xi_{\text {std }}$.

A curve $\gamma \subset(M, \xi)$ is called Legendrian if for all $p \in \gamma$ and $\xi_{p}$ the contact plane at $p, T_{p} \gamma \subset \xi_{p}$.

Throughout the rest of this section we will work with Legendrian knots in $\left(\mathbb{R}^{3}, \xi_{\text {std }}\right)$. Here Legendrian curves, in particular knots and links, are studied via projections and a common projection is the front projection (on $x z$-plane). The Legendrian condition implies that $y=d z / d x$ (the $y$-coordinate can be recovered as the slope in the $x z-$ plane). Therefore, front projections of Legendrian knots do not have vertical tangencies. Figure 1 shows two front projections of Legendrian unknots and that of a Legendrian right-handed trefoil. Since the positive $y$-axis points inside the page, at each crossing the overstrand is always the one with smaller slope.

Apart from the topological knot class, there are two classical invariants of Legendrian knots, the Thurston-Bennequin number (tb) and the rotation number (rot). The Thurston-Bennequin number measures the amount of twisting of the contact planes 

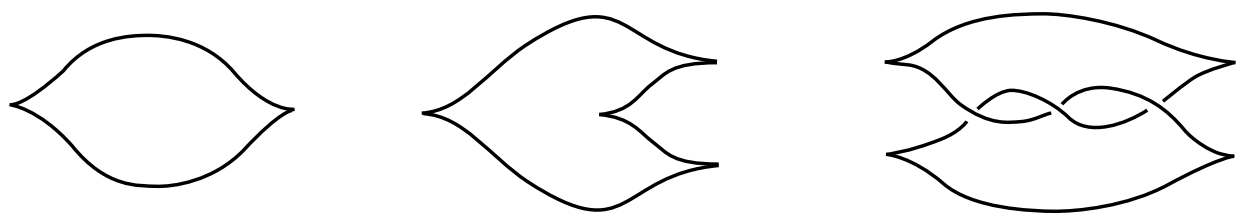

Figure 1. Legendrian unknots and Legendrian right-handed trefoil

along the knot and it does not depend on the chosen orientation of $K$. To compute the Thurston-Bennequin number of a Legendrian knot $K$, consider a nonzero vector field $v$ transverse to $\xi$, take $K^{\prime}$ the push-off of $K$ in the direction of $v$ and define $\operatorname{tb}(K):=\operatorname{lk}\left(K, K^{\prime}\right)$. The invariant $\operatorname{tb}(K)$ measures the twisting of the contact framing on $K$ with respect to the Seifert framing. For a Legendrian knot $K, \operatorname{tb}(K)$ can be computed from its front projection $\widetilde{K}$ as

$$
\operatorname{tb}(K)=\operatorname{writhe}(\tilde{K})-\frac{1}{2} \# \operatorname{cusps}(\tilde{K}) .
$$

To define the rotation number, $\operatorname{rot}(K)$, assume $K$ is oriented and $K=\partial \Sigma$, where $\Sigma \subset \mathbb{R}^{3}$ is an embedded oriented surface. When restricted to $\Sigma$, the contact planes form a trivial 2-dimensional bundle and the trivialization of $\left.\xi\right|_{\Sigma}$ induces a trivialization on $\left.\xi\right|_{K}=K \times \mathbb{R}^{2}$. Let $v$ be a nonzero vector field tangent to $K$ pointing in the direction of the orientation on $K$. The winding number of $v$ about the origin with respect to this trivialization is the rotation number of $K, \operatorname{rot}(K)$. The vector fields $d_{1}=\partial / \partial y$ and $d_{2}=-y(\partial / \partial z)-\partial / \partial x$ define a positively oriented trivialization for $\xi_{\text {std }}$. Therefore, $\operatorname{rot}(K)$ is given by the signed count ( + for counterclockwise and - for clockwise) of how many times the positive tangent vector to $K$ crosses $d_{1}$ as we travel once around $K$. The tangent vector aligns with one of the vectors $d_{1}$ or $-d_{1}$ at the points corresponding to cusps in the front projection, $\widetilde{K}$, and one can check that

$$
\operatorname{rot}(K)=\frac{1}{2}(\# \text { down cusps }-\# \text { up cusps })(\tilde{K}) .
$$

Given a Legendrian knot $K$, Legendrian knots in the same topological class as $K$ can be obtained by stabilizations. A stabilization means replacing a strand of $K$ in the front projection of $K$ by one of the zigzags in Figure 2. The stabilization is said to be positive if down cusps are introduced and negative if up cusps are introduced. The Legendrian isotopy type of $K$ changes through stabilization and so do the Thurston-Bennequin number and rotation number: $\operatorname{tb}\left(S_{ \pm}(K)\right)=\operatorname{tb}(K)-1$ and $\operatorname{rot}\left(S_{ \pm}(K)\right)=\operatorname{rot}(K) \pm 1$.

Eliashberg [4] proved that if $K$ is a Legendrian knot in $\left(\mathbb{R}^{3}, \xi_{\text {std }}\right)$ and $\Sigma$ is a Seifert surface for $K$, then

$$
\operatorname{tb}(K)+|\operatorname{rot}(K)| \leq-\chi(\Sigma) .
$$



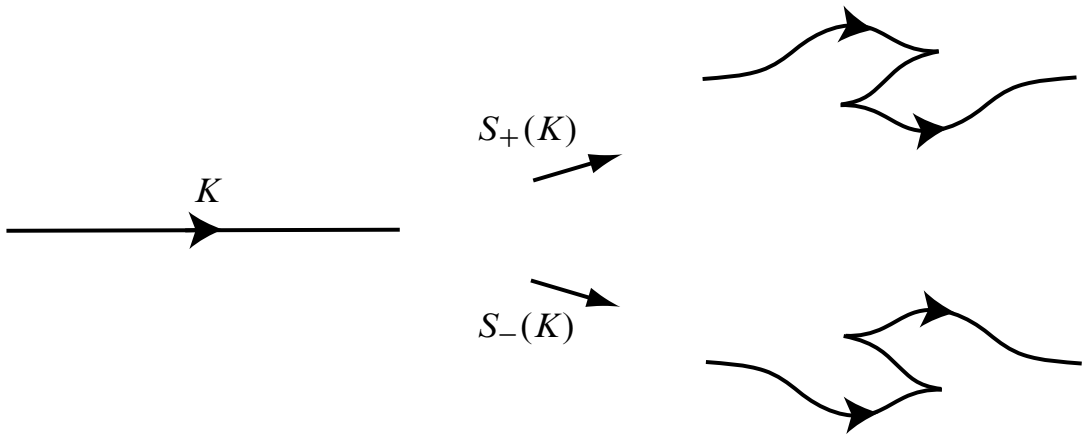

Figure 2. Positive and negative stabilizations in the front projection

In particular, if $K$ is the unknot, then $\operatorname{tb}(K) \leq-1$. If $T$ is the right-handed trefoil, then $\operatorname{tb}(T) \leq 1$. Both inequalities are sharp and equalities are realized for the first and third knots in Figure 1.

\section{Legendrian graphs}

Definition 3.1 A Legendrian graph in a contact 3-manifold $(M, \xi)$ is a graph embedded in such a way that all its edges are Legendrian curves that are nontangent to each other at the vertices. This will also be referred to as a Legendrian embedding of $G$ or a Legendrian realization of $G$, for a given graph $G$.

It should be noted that, for Legendrian graphs, if all edges around a vertex are oriented outward, then no two tangent vectors at the vertex coincide in the contact plane. However, two tangent vectors may have the same direction but different orientations resulting in a smooth arc through the vertex. It is a result of this structure that the order of the edges around a vertex in a contact plane is not changed up to cyclic permutation under Legendrian isotopy.

Theorem 3.2 Given any embedded graph $G$ in $\mathbb{R}^{3}$ there exists a Legendrian realization of $G$ in $\left(\mathbb{R}^{3}, \xi_{\text {std }}\right)$.

Proof Denote the vertices of $G$ by $v_{1}, v_{2}, \ldots, v_{n}$, and fix these points. Every point $v_{i}$ has an $\epsilon$-neighborhood $U_{i}$ contactomorphic to a neighborhood of the origin in $\left(\mathbb{R}^{3}, \xi_{\text {sym }}\right)$, in cylindrical coordinates $(r, \theta, z)$. Via this diffeomorphism, the contact plane $\xi_{v_{i}}$ is identified with the plane $z=0$ at the origin. Near each vertex $v_{i}$, we modify $G$ through ambient isotopy such that the edges incident with $v_{i}$ are segments 
which lie in the contact plane $\xi_{v_{i}}$ and are identified with $\theta$-constant segments in the plane $z=0$. The edges of $G$ are thus Legendrian near each vertex.

Consider an edge $e$ between two vertices $v_{i}$ and $v_{j}$ and let $e$ be identified with the $\theta_{i}-$ ray near $v_{i}$ and with the $\theta_{j}$-ray near $v_{j}$. Denote by $p_{i} \in U_{i}$ and $p_{j} \in U_{j}$ the two points which are identified with $\left(\epsilon / 2, \theta_{i}, 0\right)$ and $\left(\epsilon / 2, \theta_{j}, 0\right)$. Denote by $e_{p_{i}}$ the Legendrian segment between $v_{i}$ and $p_{i}$ identified with the segment $0 \leq r \leq \epsilon / 2, \theta=\theta_{i}, z=0$, and by $e_{p_{j}}$ the Legendrian segment between $v_{j}$ and $p_{j}$ identified with the segment $0 \leq r \leq \epsilon / 2, \theta=\theta_{j}, z=0$. Denote by $e_{i j}$ the arc of $e$ between $p_{i}$ and $p_{j}$. We can $C^{0}$-approximate $e_{i j}$ by a Legendrian arc $\widetilde{e_{i j}}$, in such a way that the union of arcs $e_{p_{i}} \cup \widetilde{e_{i j}} \cup e_{p_{j}}$ is a $C^{1}$-curve. We do this by choosing a $C^{0}$-close approximation of the front projection of $e_{i j}$ by a regular curve $\overline{e_{i j}}$ with no vertical tangencies, with isolated cusps and such that at each point $p \in \overline{e_{i j}}$ the slope of $\overline{e_{i j}}$ is close to the $y$-coordinate of the point on $e_{i j}$ projecting to $p$. We can do this while keeping the endpoints $p_{i}$ and $p_{j}$ fixed and in such a way that the Legendrian lift of $\overline{e_{i j}}$ to $\widetilde{e_{i j}}$ coincides with $e$ near $p_{i}$ and $p_{j}$; see Geiges [7, Section 3.3.1]. The curve $e_{p_{i}} \cup \widetilde{e_{i j}} \cup e_{p_{j}}$ is a Legendrian curve which is $C^{0}$-close to $e$.

We approximate all other edges in the same way, and we obtain a Legendrian $C^{0}$ approximation of $G$ (ie, a Legendrian graph topologically ambient isotopic to $G$ ).

Similar to Legendrian knots, Legendrian graphs can be studied via front projections. Two generic front projections of a Legendrian graph are related by Reidemeister moves I, II and III together with three moves given by the mutual position of vertices and edges; see Baader and Ishikawa [1]. See Figure 3.

The order of edges around a vertex in the front projection is not the same as the order in the contact plane. Through Legendrian isotopy, the order of the edges around a vertex in the contact plane does not change but the order in the front projection may change (see move VI).

We extend the classical invariants tb and rot to Legendrian graphs. A cycle in a Legendrian graph is a piecewise smooth Legendrian knot, a simple closed curve that is everywhere tangent to the contact planes but has finitely many points (the vertices) where it may not be smooth. We first define the invariants tb and rot for piecewise smooth Legendrian knots, then we extend the definition to Legendrian graphs.

Given any piecewise smooth Legendrian knot $K$, there is a natural way to construct a smooth knot from $K$. We define the standard smoothing of a cycle $K$ of a Legendrian graph to be the $C^{1}$-curve obtained by replacing $K$ in an $\epsilon$-neighborhood of each vertex by the lift of a minimal front projection of a smooth curve, which coincides with $K$ outside of the $\epsilon$-neighborhood. The projection is minimal in the sense that no 


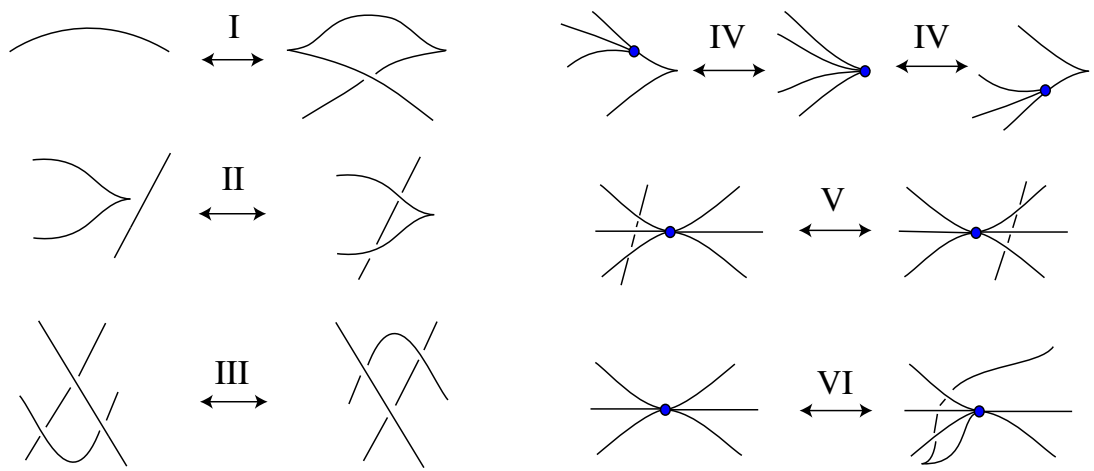

Figure 3. Legendrian isotopy moves for graphs: Reidemeister moves I, II and III, a vertex passing through a cusp (IV), an edge passing under or over a vertex (V), an edge adjacent to a vertex rotates to the other side of the vertex (VI). Reflections of these moves that are Legendrian front projections are also allowed.

extra stabilizations or knotting are introduced with this approximation. See Figure 4. We denote the standard smoothing of a piecewise smooth Legendrian knot $K$ by $K_{\text {st }}$.
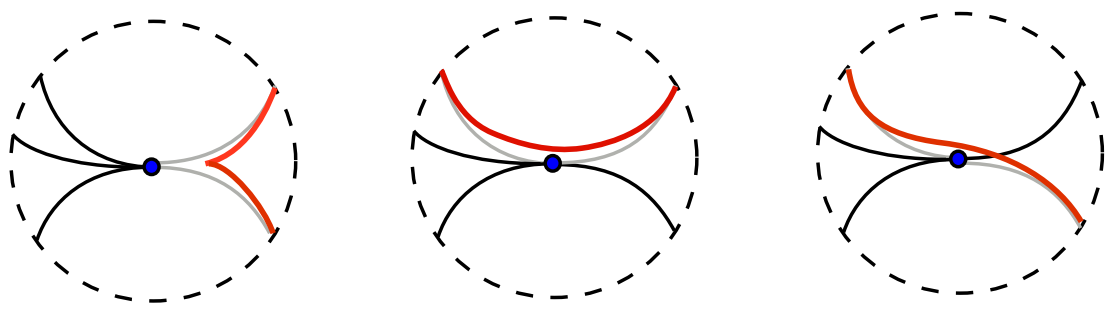

Figure 4. Approximation by a $C^{1}$-curve near a vertex. The grey edges which are part of the cycle $K$ are replaced by the bold arc $C^{1}$-arc near the vertex.

The uniqueness of this construction is proved by the following theorem.

Theorem 3.3 If $K_{1}$ and $K_{2}$ are piecewise smooth Legendrian knots which are isotopic as Legendrian graphs, then their standard smoothings are isotopic Legendrian knots.

Proof Since $K_{1}$ and $K_{2}$ are isotopic as Legendrian graphs, their front projections, $\widetilde{K_{1}}$ and $\widetilde{K}_{2}$, are related by a finite sequence of Reidemeister moves I-VI and Legendrian planar isotopy. Thus to show that the standard smoothings of $K_{1}$ and $K_{2}$ are isotopic 
we need only show that the standard smoothings of valence two subgraphs of the Reidemeister moves IV, V and VI result in isotopic Legendrian knots.

For Reidemeister move IV, there are two different possibilities of valence two subgraphs up to reflection and planar isotopy to be considered; see Figure 5(a). After smoothing, the move either shows no change or a planar isotopy.

For Reidemeister move V, there are two different possibilities of valence two subgraphs up to reflection and planar isotopy to be considered; see Figure 5(b). After smoothing, the move either shows a planar isotopy or a difference of a Reidemeister move II.

For Reidemeister move VI, there are three different possibilities of valence two subgraphs up to reflection and planar isotopy to be considered; see Figure 5(c). After smoothing, the move either shows no change, a planar isotopy, or a difference of a Reidemeister move I.

(a)
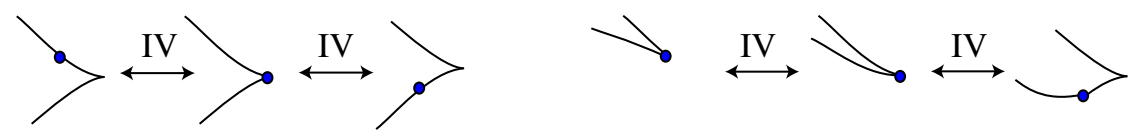

(b)
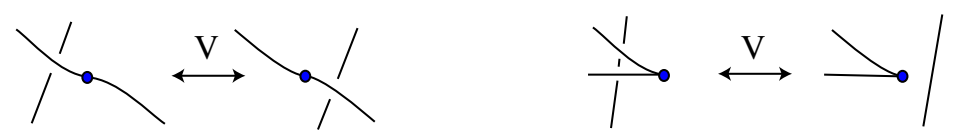

(c)
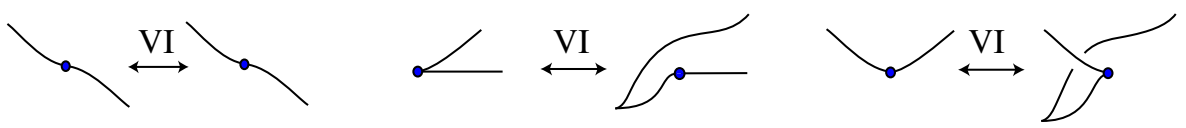

Figure 5. Subdiagrams of Legendrian graphs moves

Of course, for any piecewise smooth Legendrian knot there are isotopic Legendrian knots which can be obtained by a small isotopy near each vertex moving the edges so that they have parallel tangents at the vertex. In light of Theorem 3.3, we have the following:

Corollary 3.4 The isotopy class of a piecewise smooth Legendrian knot contains Legendrian embeddings from exactly one isotopy class of a Legendrian knot, the knot obtained by its standard smoothing.

This parallels the relationship between knots in the piecewise linear category and knots in the smooth category. 
Given a piecewise smooth Legendrian knot $K$ and its standard smoothing $K_{\text {st }}$,one could take the definition of the classical invariants to be $\operatorname{tb}(K)=\operatorname{tb}\left(K_{\mathrm{st}}\right)$ and $\operatorname{rot}(K)=$ $\operatorname{rot}\left(K_{\mathrm{st}}\right)$. However, since we will be using the said definitions to define invariants for Legendrian graphs, we would like to define them in such a way that smoothings are not needed. It should be noted that any other definition of tb and rot that coincides with the invariants for smooth Legendrian knots will be equivalent to the above definition, as a result of Theorem 3.3.

\subsection{The Thurston-Bennequin number}

Let $K$ represent a piecewise smooth Legendrian knot and $v$ a vector field along $K$ which is transverse to the contact planes. Let $K^{\prime}$ be the push-off of $K$ in the direction of $v$ and let $\operatorname{tb}(K):=\operatorname{lk}\left(K, K^{\prime}\right)$. Since the change in direction at the singular points occurs in the contact plane, a transverse push-off is still well-defined. This definition coincides with that for smooth knots.

Definition 3.5 For a Legendrian graph $G$, fix an order on the cycles of $G$ and define the Thurston-Bennequin number of $G$, denoted by $\operatorname{tb}(G)$, to be the ordered list of the Thurston-Bennequin numbers of the cycles of $G$. If $G$ has no cycles, define $\operatorname{tb}(G)$ to be the empty list.

\subsection{The rotation number}

We define the rotation number of a piecewise smooth Legendrian knot $K \subset\left(\mathbb{R}^{3}, \xi_{\text {std }}\right)$ as follows: Consider an embedded oriented surface $\Sigma \subset \mathbb{R}^{3}$ with $\partial \Sigma=K$ and endow $K$ with the orientation induced by that on $\Sigma$. Consider the trivialization of $\xi_{\text {std }}$ given by the two vectors $d_{1}=\partial / \partial y$ and $d_{2}=-y(\partial / \partial z)-\partial / \partial x$. Denote by $v_{1}, v_{2}, \ldots, v_{s}, v_{s+1}=v_{1}$ the vertices on $K$, in cyclic order as given by the orientation. Denote by $e_{i}$ for $i=1, \ldots, s$ the edge of $K$ between $v_{i}$ and $v_{i+1}$, and denote by $T_{i}$ for $i=1, \ldots, s$ the unit vector field tangent to $e_{i}$ pointing in the direction of the orientation on $K$. We follow $T_{i}$ in the trivialization given by $d_{1}$ and $d_{2}$ and count with sign the number of times $T_{i}$ passes $d_{1}$. At each vertex $v_{i}, i=2, \ldots, s+1$, if $T_{i-1}$ and $T_{i}$ do not coincide, we complete the rotation counterclockwise if $\left\{T_{i-1}, T_{i}\right\}$ is a positively oriented basis for $\xi_{v_{i}}$ and clockwise if $\left\{T_{i-1}, T_{i}\right\}$ is a negatively oriented basis for $\xi_{v_{i}}$. This is equivalent to completing by a rotation from $T_{i-1}$ towards $T_{i}$ in the direction of the shortest angle between them. Note that since one edge is oriented towards the vertex and one edge is oriented away from the vertex, $T_{i-1}$ and $T_{i}$ cannot be opposite to each other. 
Denote by $p(K)$ the number of times $d_{1}$ is passed in the counterclockwise direction and by $n(K)$ the number of times $d_{1}$ is passed in the clockwise direction as $K$ is traced once. We define the rotation number of $K$ by

$$
\operatorname{rot}(K):=p(K)-n(K) .
$$

Let $\mathcal{L}(K)$ denote the Legendrian isotopy class of $K$. The above discussion gives a recipe of how to compute the rotation number for a particular embedding of a piecewise smooth Legendrian knot. However, when $K$ changes through Legendrian isotopy, the tangent vector at $K$ changes continuously and the edges cannot pass over one another at the vertices. Thus we get a continuous map rot: $\mathcal{L}(K) \rightarrow \mathbb{Z}$. The rotation number is therefore a Legendrian isotopy invariant for piecewise smooth Legendrian knots. If $K$ is a smooth Legendrian knot then we recover the rotation number for $K$.

Definition 3.6 For a Legendrian graph $G$, fix an order on the cycles of $G$ with orientation and define the rotation number of $G$, denoted by $\operatorname{rot}(G)$, to be the ordered list of the rotation numbers of the cycles of $G$. If $G$ has no cycles, define $\operatorname{rot}(G)$ to be the empty list.

\section{Nonrealization of maximal tb}

A trivial unknot is one with $t b=-1$ and rot $=0$ (like the first unknot shown in Figure 1). The trivial unknot attains the maximal possible Thurston-Bennequin number for an unknot. In [11], Mohnke used the Kauffman polynomial to prove that the Borromean rings and the Whitehead link cannot be realized as Legendrian links of trivial unknots. In this section we determine which graphs can be Legendrian realized in such a way that all cycles are trivial unknots. We will see that there are many graphs, even planar graphs, with no such Legendrian realization. We give a full characterization of these graphs. We also present a more general result about which graphs can be realized with all their cycles having maximal tb for a class of knots. The following two lemmas will be useful.

Lemma 4.1 The front projections of any two Legendrian realizations of a graph in $\left(\mathbb{R}^{3}, \xi_{\text {std }}\right)$ are related by a finite sequence of Legendrian isotopies and changes of the following two types:

- replacement of one edge with a different Legendrian arc between the same vertices (changing the number of stabilizations in the edge and/or the number of crossings of the edge with itself),

- replacement of two edges (changing the number of crossings between the two edges). 
Proof If the two Legendrian realizations are Legendrian isotopic, then the two front projections differ by Legendrian isotopy only. In this case one projection can be taken to the other projection by planar isotopies and Reidemeister moves I-VI. If not, the two front projections may differ in number of edge stabilizations or number of crossings. The stabilizations occur on a single edge while the crossings may occur on a single edge or between two edges. In this case, when the realizations are not Legendrian isotopic, to take one front projection to the other replacement of one edge or a pair of edges will be needed in addition to planar isotopy and Reidemeister moves I-VI.

Lemma 4.2 For any Legendrian embedding $L$ of $K_{4}$ in $\left(\mathbb{R}^{3}, \xi_{\text {std }}\right)$,

$$
\sum_{\gamma \in \Gamma_{L}} \mathrm{tb}(\gamma) \equiv 0 \bmod 2,
$$

where $\Gamma_{L}$ is the set of cycles in $L$.

Proof Denote by $v_{1}, v_{2}, v_{3}$ and $v_{4}$ the vertices of an abstract $K_{4}$. There are seven cycles in $K_{4}$ : four 3-cycles $\left(v_{1} v_{2} v_{3}, v_{1} v_{2} v_{4}, v_{1} v_{3} v_{4}, v_{2} v_{3} v_{4}\right)$ and three 4-cycles $\left(v_{1} v_{2} v_{3} v_{4}, v_{1} v_{2} v_{4} v_{3}, v_{1} v_{3} v_{2} v_{4}\right)$. Each edge appears in four different cycles and each pair of edges appears in two different cycles. Consider the embedding $K$ of $K_{4}$ shown in Figure 6.

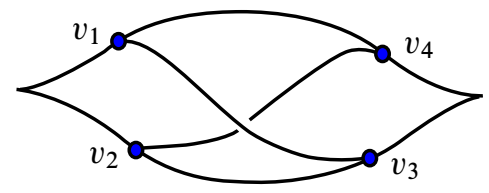

Figure 6. A Legendrian embedding of $K_{4}$

For this embedding, there are six cycles with $\mathrm{tb}=-1$ and one cycle $\left(v_{1} v_{2} v_{4} v_{3}\right)$ with $\mathrm{tb}=-2$, thus

$$
S:=\sum_{\gamma \in \Gamma_{K}} \operatorname{tb}(\gamma)=-8
$$

Take an arbitrary Legendrian embedding of $K_{4}$, call it $L$. By Lemma 4.1, the front projection for this embedding differs from $K$ by a finite sequence of Legendrian isotopies and changes of the form described above in one edge or two edges. Since tb is an invariant, $S$ does not change under isotopy.

- If a change in a single edge is made, the tb for all four cycles containing this edge is modified by the same quantity.

- If a change in two edges is made, the tb for both cycles containing this pair of edges is modified by the same quantity. 
Thus, the parity of the sum of the tb's over all cycles remains unchanged throughout the process and

$$
\sum_{\gamma \in \Gamma_{L}} \mathrm{tb}(\gamma) \equiv 0 \bmod 2
$$

We define $L_{\text {odd }}$ to be the set of topological knot classes with odd maximal ThurstonBennequin number. We have the following theorem:

Theorem 4.3 Let $G$ be a graph that contains $K_{4}$ as a minor. Then there does not exist a Legendrian realization of $G$ such that all its cycles are knots in $L_{\text {odd }}$ realizing their maximal Thurston-Bennequin number.

Proof It suffices to prove the theorem for $G=K_{4}$, since any graph that contains $K_{4}$ as a minor contains a subdivision of $K_{4}$ (see Definition 4.6). Assume all seven cycles of $K_{4}$ can be realized with odd maximal Thurston-Bennequin number, $2 t_{n}+1$, $n=1, \ldots, 7$. Then $\sum_{n=1}^{7}\left(2 t_{n}+1\right) \not \equiv 0 \bmod 2$, contradicting the conclusion of Lemma 4.2.

Since the unknot has odd maximal tb $=-1$ we obtain the following corollary. Recall that the trivial unknot is the unknot with maximal Thurston-Bennequin number.

Corollary 4.4 Let $G$ be a graph that contains $K_{4}$ as a minor. Then there does not exist a Legendrian realization of $G$ such that all its cycles are trivial unknots.

When we consider a graph $G$ that contains $K_{4}$ as a minor and an arbitrary Legendrian embedding $f(G)$,Corollary 4.4 guarantees either $f(G)$ contains a nontrivial knot or that all cycles of $f(G)$ are unknots but they do not all have maximal tb. For any intrinsically knotted graph (see Section 2.1) Corollary 4.4 is not surprising, since every embedding of an intrinsically knotted graph contains a nontrivial knot. However, those graphs that contain $K_{4}$ as a minor include some planar graphs and all nonplanar graphs. Corollary 4.4 is most impressive for those planar graphs but also adds to our understanding of all those graphs which are nonplanar and not intrinsically knotted.

Here we outline the implications of Theorem 4.3. Given an arbitrary Legendrian embedding $f(G)$ of a graph $G$ that contains $K_{4}$ as a minor, Theorem 4.3 implies that either $f(G)$ does not contain knots exclusively from $L_{\text {odd }}$ or $f(G)$ contains only knots from $L_{\text {odd }}$ and they do not all attain their maximal tb. So Theorem 4.3 gives more information about intrinsically knotted graphs. One example of an intrinsically knotted graph is $K_{7}$; see Conway and Gordon [2]. There are embeddings of $K_{7}$ with unknots as all but one cycle which is a trefoil. Both the unknot and the right-handed 
trefoil have odd maximal Thurston-Bennequin number, so Theorem 4.3 implies that for such a Legendrian embedding not all cycles attain their maximal tb. There are many other knots whose maximal Thurston-Bennequin number is odd. Additional examples are the figure eight knot, the $6_{1}$ knot and its mirror image and the $6_{2}$ knot and its mirror image.

Remark 4.5 There is a more general version of Theorem 4.3 that is an immediate consequence of the proof, though it is cumbersome to state. Let $G$ be a graph that contains $K_{4}$ as a minor. Let $S$ be a subdivision of $K_{4}$ contained in $G$. Then there does not exist a Legendrian realization of $G$ such that $S$ contains precisely an odd number of cycles that are knots in $L_{\text {odd }}$ realizing their maximal Thurston-Bennequin number.

If we focus on embeddings with only unknots as in Corollary 4.4, the converse also holds. It is worth noting, since the set of intrinsically knotted graphs is a subset of nonplanar graphs and $K_{4}$ is planar, that all of those graphs that do not contain $K_{4}$ as a minor are also planar. Thus there is no obvious obstruction to the converse of Corollary 4.4. Before proving the converse we introduce some needed definitions and observations.

Definition 4.6 (1) A path between two vertices $v_{1}$ and $v_{2}$ of a graph $G$ is a finite sequence of at least two edges starting at $v_{1}$ and ending at $v_{2}$, with no repetition of vertices.

(2) A vertex of the graph $G$ is said to be a cut vertex if by deleting the vertex (and all incident edges) the resulting graph has more connected components than $G$.

(3) An edge of $G$ is said to be a cut edge if by deleting the edge the resulting graph has more connected components than $G$.

(4) A subdivision of the graph $G$ is a graph obtained by replacing a finite number of edges of $G$ with paths (one can think of this as adding a finite number of vertices along edges of $G$ ).

Remark 4.7 Let $G$ be a graph which does not contain $K_{4}$ as a minor. Let $v_{1}, v_{2}$, $v_{3}$ and $v_{4}$ be four vertices in a cycle of $G$, appearing in this order. For any such formation, there are not two edges or paths, other than the ones already contained in the cycle, connecting $v_{1}$ and $v_{3}$ and connecting $v_{2}$ and $v_{4}$. Otherwise $v_{1}, v_{2}, v_{3}$ and $v_{4}$ represent the vertices of a (subdivision of) $K_{4}$. See Figure 7(a). 
Remark 4.8 Let $G$ be a graph which does not contain $K_{4}$ as a minor. Let $v_{1}, v_{2}$ and $v_{3}$ be three vertices in a cycle of $G$. For any such formation, there is not an additional vertex $v$ with distinct edges (or paths that only intersect in $v$ ) connecting $v$ to the vertices $v_{1}, v_{2}$ and $v_{3}$. Otherwise $v_{1}, v_{2}, v_{3}$ and $v$ represent the vertices of a (subdivision of) $K_{4}$. See Figure 7(b).

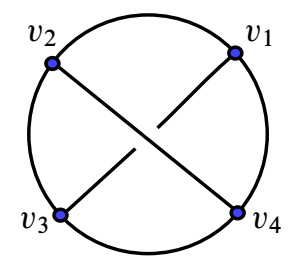

(a)

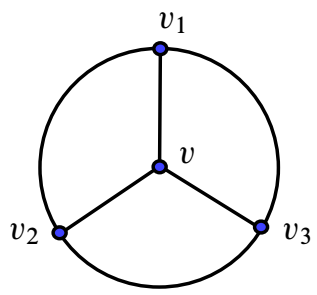

(b)

Figure 7. Embeddings of $K_{4}$ as described in Remarks 4.7 and 4.8

Theorem 4.9 If $G$ is a graph that does not contain $K_{4}$ as a minor, then it can be Legendrian realized in $\left(\mathbb{R}^{3}, \xi_{\text {std }}\right)$ in such a way that all its cycles are trivial unknots.

Proof We need only prove the theorem for $G$ connected, with no cut edges and no cut vertices. In all other cases such components (connected components with no cut edges nor cut vertices) of $G$ can be realized in the same way and cut edges can be realized in any fashion (as they do not appear in any cycle).

In what follows, all edges of $G$ are realized as nonstabilized arcs. Let $C$ be one of the cycles of $G$ and $s$ the number of vertices of $C$. Realize $C$ in such a way that its front projection consists of $s-1$ horizontal edges and one edge on top of these. Label the vertices on $C$ with $v_{1}, v_{2}, \ldots, v_{s}$, in this order, from left to right. Realize all the other edges between vertices of $C$ on top of the horizontal edges of $C$, in a nested fashion as shown in Figure 8(a). By Remark 4.7, we can realize these edges without any crossings. Otherwise, the four endpoints of two crossing edges represent the vertices of a (subdivision of) $K_{4}$.

Next, for each pair of vertices $\left(v_{i}, v_{j}\right), i<j$, of $C$ for which there exists at least one path between $v_{i}$ and $v_{j}$ not containing any additional vertices of $C$, realize one of these paths under the horizontal line of $C$. Call this path $P_{i j}^{1}$. For each $t>1$, if there is another path between $v_{i}$ and $v_{j}$ not containing additional vertices of $C$ or $P_{i j}^{1}, \ldots, P_{i j}^{t-1}$, realize this path under $P_{i j}^{t-1}$. See Figure 8(b). Let this set of paths between $v_{i}$ and $v_{j}$ be denoted by

$$
\mathcal{P}_{i j}:=\left\{P_{i j}^{1}, P_{i j}^{2}, \ldots, P_{i j}^{t}, \ldots\right\} .
$$

This is a finite set. 
By construction, all paths in $\mathcal{P}_{i j}$ are nested, without any crossings, they have the vertices $v_{i}$ and $v_{j}$ in common and are disjoint otherwise. By Remark 4.7, we can realize all the paths appearing thus far without any crossings.

Additionally, for any vertices $v_{i}, v_{j}, v_{k}$ and $v_{l}$ of $C$, with $\left(v_{i}, v_{j}\right) \neq\left(v_{k}, v_{l}\right)$, no element of $\mathcal{P}_{i j}$ has any vertices (distinct from $v_{i}, v_{j}, v_{k}$ and $v_{l}$ ) or edges in common with any element of $\mathcal{P}_{k l}$. We prove the observation by contradiction. There are four different cases of $\left(v_{i}, v_{j}\right) \neq\left(v_{k}, v_{l}\right)$, that is, (1) $i=k$ and $j \neq l,(2) i \neq k$ and $j=l$, (3) $j=k$ and (4) $i, j, k$ and $l$ are all distinct. Suppose a path $P$ in $\mathcal{P}_{i j}$ and a path $Q$ in $\mathcal{P}_{k l}$ have at least one vertex other than $v_{i}, v_{j}, v_{k}$ and $v_{l}$ in common. Let $\mathcal{V}$ be the nonempty set of vertices that the paths $P$ and $Q$ have in common, distinct from $v_{i}$, $v_{j}, v_{k}$ and $v_{l}$. For the subcases (1), (3) and (4), let $w \in \mathcal{V}$ be the vertex in this set closest to $v_{l}$ on the path $Q$. Then the part of the path $Q$ from $w$ to $v_{l}$ is distinct from the path $P$. So this path between $w$ and $v_{l}$ together with the two parts of $P$ going from $v_{i}$ to $w$ and from $w$ to $v_{j}$ are all paths joining $w$ to the cycle $C$ which only intersect in the endpoint $w$. Thus by Remark 4.8, we have a contradiction. Similarly, for case (2), let $u \in \mathcal{V}$ be the vertex in this set closest to $v_{i}$ on the path $P$. Then the part of the path $P$ from $u$ to $v_{i}$ together with the two parts of $Q$ going from $v_{k}$ to $u$ and from $u$ to $v_{l}$ are all paths joining $u$ to the cycle $C$ which only intersect in the endpoint $u$. Thus by Remark 4.8 we have a contradiction.

Further, we treat each of the elements in $\mathcal{P}_{i j}$, for all $i, j, 1 \leq i<j \leq s$, as if it were the path of horizontal edges in $C$. We realize the edges with vertices on each such path above the path and the paths with endpoints on each such path below the path. We continue to realize each new edge on top of the path its vertices lie on and each new path below the path its vertices lie on.

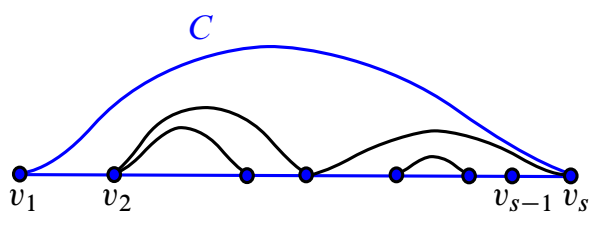

(a)

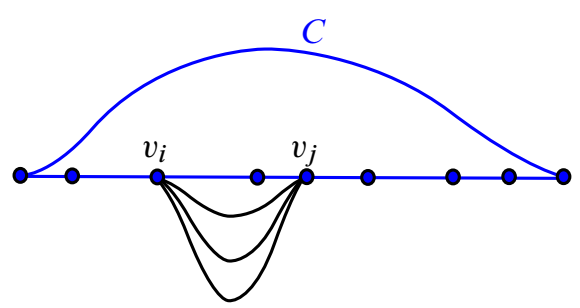

(b)

Figure 8. First steps in Legendrian realizing $G$ with all cycles unknots of maximal tb. The cycle $C$ is blue.

Claim (1) This is a Legendrian embedding of $G$ (ie all edges and vertices of $G$ are realized).

(2) All cycles in this embedding have maximal tb $=-1$. 
Proof of (1) The above construction is a prescription for a front projection, thus as long as all vertices and edges of $G$ are realized we have a Legendrian embedding. The two cases of assuming a vertex was not realized and assuming an edge was not realized will be treated concurrently. If there exists an edge of $G$ which has not been realized, then there is an edge or a path in $G$ between two realized vertices that has not been realized. If there exists a vertex of $G$ which has not been realized, then, since $G$ was assumed without cut edges and connected, there is a path in $G$ between two realized vertices that has not been realized. We show that the existence of such an edge or path leads to a contradiction.

Let $v$ and $w$ represent two realized vertices of $G$ such that there is a nonrealized edge or path (containing exclusively edges that have not been realized) between $v$ and $w$. The vertices $v$ and $w$ cannot both be in $C$, since all paths and edges between these vertices have been realized. For the same reason, $v$ and $w$ cannot both be vertices on the same element of a $\mathcal{P}_{i j}$ or on the same path realized at a later stage.

From the vertex $w$ we will form a cycle $\mathcal{C}_{w}$ in the realized projection. The cycle $\mathcal{C}_{w}$ consists of the path to the right of $w$ formed by choosing the edge that is to the right and upper most at each vertex (this path will connect $w$ with $v_{s}$ ), the path to the left of $w$ formed by taking the edge that is to the left and upper most at each vertex (this path will connect $w$ with $v_{1}$ ) and the edge connecting $v_{1}$ and $v_{s}$. The vertex $v$ is not on the cycle $\mathcal{C}_{w}$, or we would have realized a path between $v$ and $w$. Now let $\mathcal{P}_{x}$ be the path in the realized projection formed by starting at $v$ and choosing the edge that is to the right and upper most at each vertex until the vertex is a vertex of $\mathcal{C}_{w}$. Call this vertex $x$. Let $\mathcal{P}_{y}$ be the path in the realized projection formed by starting at $v$ choosing the edge that is to the left and upper most at each vertex until the vertex is a vertex of $\mathcal{C}_{w}$. Call this vertex $y$. The vertices $w, x$ and $y$ are all on the cycle $\mathcal{C}_{w}$, the (edges or) paths $\mathcal{P}_{x}, \mathcal{P}_{y}$ and the unrealized edge or path from $v$ to $w$ are all disjoint away from $v$, thus by Remark 4.8, this cannot occur. Therefore there is no such unrealized edge or path.

Proof of (2) Since the construction does not contain any crossings, we need only show that no cycle exhibits a stabilization. Assume there is a cycle in the embedding which represents a stabilized unknot. Since all edges were realized as nonstabilized arcs in the first place, at each cusp of this unknot there is a vertex of $G$. In the stabilized unknot there are at least two left cusps and two right cusps. We denote the vertices at the left cusps by $w_{1}$ and $w_{3}$ and the vertices at the right cusps by $w_{2}$ and $w_{4}$, with the four vertices appearing in order $w_{1}, w_{2}, w_{3}, w_{4}$ in the cycle. Without loss of generality we may assume that either $w_{2}, w_{3}$ and $w_{4}$ are at consecutive right-left-right cusps and $w_{1}$ is on the left of $w_{3}$, as in Figure 9(a), or $w_{1}, w_{4}$ and $w_{3}$ are at consecutive 
left-right-left cusps and $w_{2}$ is on the right of $w_{4}$, as in Figure 9(b). We show that a $K_{4}$ necessarily exists.

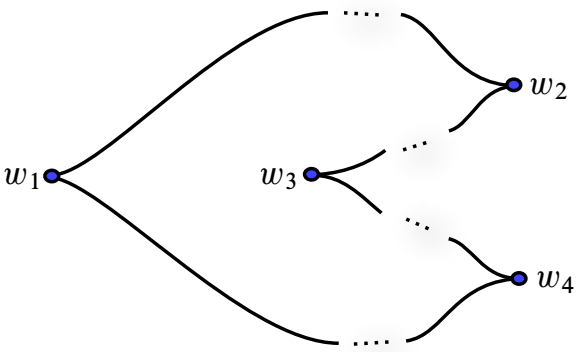

(a)

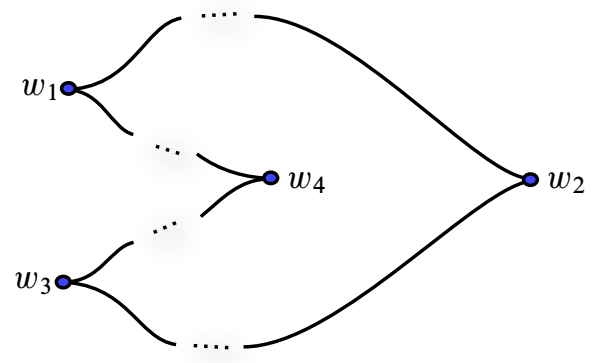

(b)

Figure 9. Cycle representing a stabilized unknot

We prove the case pictured in Figure 9(a). The other case is similar. We consider a path $P_{2}$ which starts at $w_{2}$ and always follows along an edge to the right at each vertex. We also consider a path $P_{4}$ which starts at $w_{4}$ and always follows along an edge to the right at each vertex. Denote the first vertex where $P_{2}$ and $P_{4}$ intersect by $u$. The vertex $u$ may be $w_{2}, w_{4}, v_{s}$ or a point in between. The path which follows $P_{2}$ from $w_{2}$ to $u$ and then $P_{4}$ from $u$ to $w_{4}$ is a path between $w_{2}$ and $w_{4}$ which is disjoint from both $w_{1}$ and $w_{3}$, since both $w_{1}$ and $w_{3}$ lie on the left of both $w_{2}$ and $w_{4}$. This is a third path between $w_{2}$ and $w_{4}$, in addition to the two included in the stabilized cycle. Now consider a path $P_{3}$ which starts at $w_{3}$ and always follows along an edge to the left at each vertex. The path $P_{3}$ will eventually reach $v_{1}$ and will intersect the stabilized cycle in a vertex $u^{\prime}$ situated on the arc of the cycle which goes between $w_{2}$ and $w_{4}$ and does not contain $w_{3}$. The vertices $u^{\prime}, w_{2}, w_{3}$ and $w_{4}$ are the vertices of a (subdivision of) $K_{4}$.

Remark 4.10 Recall that a graph $G$ is minor minimal with respect to a property if $G$ has the property but no minor of $G$ has the property. Corollary 4.4 together with Theorem 4.9 show that $K_{4}$ is minor minimal with respect to the property of not having a Legendrian embedding with all cycles trivial unknots. Not only that, but Theorem 4.9 shows that $K_{4}$ is the only graph in this minor minimal set, thus characterizing this property.

\section{Legendrian graphs classified by classical invariants}

It is known that certain types of Legendrian knots and links are determined by the classical invariants tb and rot in $\left(\mathbb{R}^{3}, \xi_{\text {std }}\right)$. Eliashberg and Fraser [5] showed that the 
Legendrian unknot is determined by tb and rot. Etnyre and Honda [6] showed the same holds for torus knots and the figure eight knot. Ding and Geiges [3] showed that links consisting of an unknot and a cable of that unknot are classified by their oriented link type and the classical invariants in $\left(\mathbb{R}^{3}, \xi_{\text {std }}\right)$.

In this section we investigate what types of spatial graphs are classified up to Legendrian isotopy by the pair (tb, rot). It is useful to recall that within each Legendrian isotopy class the cyclic order of the edges around a vertex in the contact plane is not changed. For now we consider graphs that have cut edges or cut vertices.

Remark 5.1 Let $G$ be a graph containing a vertex $v$ of valence at least three which is incident to at least one cut edge, $e$. For different Legendrian realizations of $G$, the order of edges at vertex $v$ can differ while the classical invariants for all cycles are the same. This is because the cut edge $e$ does not appear in any cycle. See Figure 10(a). That is, a Legendrian embedding of $G$ is not determined by the pair (tb, rot).

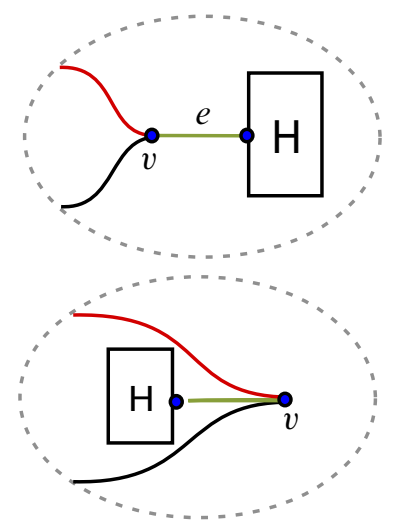

(a)
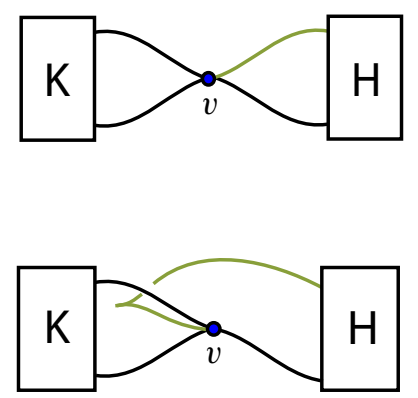

(b)

Figure 10. (a) Two realizations of a graph with a valence three vertex $v$ adjacent to one cut edge $e$ where the order of edges around vertex $v$ is not the same above as below. (b) Two realizations of a graph with a valence four cut vertex $v$ where the order of edges around vertex $v$ is not the same above as below.

Remark 5.2 Let $G$ be a graph containing a cut vertex of valence at least four. For different Legendrian realizations of $G$, the order of edges at this vertex can differ while the classical invariants for all cycles are the same. An example is shown in Figure 10(b). Note that there are no cycles containing edges from both $K$ and $H$, thus tb and rot are the same for the two embeddings. That is, a Legendrian embedding of $G$ is not determined by the pair (tb, rot). 
This means that even uncomplicated graphs carry more information as a whole than the set of knots represented by their cycles. The above remarks can be summarized into the following:

Theorem 5.3 No graph containing at least one cycle and at least one cut edge or one cut vertex is determined up to Legendrian isotopy by the pair (tb, rot).

Proof Suppose $G$ has at least one cut edge or vertex and is determined by the pair (tb, rot). Suppose $G$ has a cut vertex $v$. By Remark 5.2, the vertex $v$ must have valence 3 or less. If $v$ is a cut vertex with valence 3 , then it must be adjacent to a cut edge and Remark 5.1 implies that $G$ is not determined by the pair (tb, rot). Therefore, $v$ must have valence 2 or less. However, to be a cut vertex, $v$ must have at least valence two. For a valence 2 cut vertex both incident edges are cut edges, so by Remark 5.1 both vertices defining these edges must have valence 2 or less. Therefore, the only such graph with a cut vertex is a path graph. So $G$ has no cycles.

Suppose $G$ has a cut edge $e$ with no cut vertices. By Remark 5.1, the valences of the two vertices must be 2 or less. Since the two vertices cannot be cut vertices, they are of valence one. Thus the graph $G$ is a single edge. Therefore, there does not exist a graph containing at least one cycle and at least one cut edge or one cut vertex that is determined by the pair (tb, rot).

Next, we focus on Legendrian embeddings of the lollipop graph and the handcuff graph. See Figure 11. Both these graphs have one cut edge. For any topological class of these graphs, the Legendrian class cannot be determined by the pair (tb, rot), by Theorem 5.3.

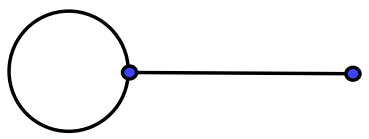

(a)

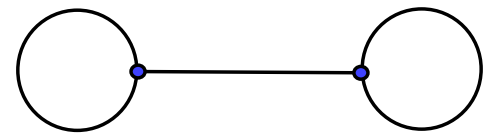

(b)

Figure 11. (a) The lollipop graph (b) The handcuff graph

Definition 5.4 A planar spatial graph is a spatial graph which is ambient isotopic to an embedding in the plane. A Legendrian planar graph (or a planar Legendrian realization) is a Legendrian realization of a planar spatial graph.

We show that, if we restrict to planar spatial graphs, a pair (tb, rot) determines exactly two Legendrian isotopy classes of the lollipop graph and a pair (tb, rot) determines exactly four Legendrian isotopy classes of the handcuff graph. We do this by constructing a Legendrian isotopy between an arbitrary embedding and a standard form embedding. 
Eliashberg and Fraser [5] showed that a Legendrian unknot $K$ is Legendrian isotopic to a unique unknot in standard form. A Legendrian unknot is in standard form if it is the lift of a front projection as in Figure 12(a) or (b). The front projection in Figure 12(a) represents two distinct Legendrian classes, depending on the chosen orientation. For the front projection shown in Figure 12(b) both orientations give the same Legendrian class. Fix the orientation to be the one which makes the left cusp a down cusp. The number of cusps and crossings of the unknot in standard form are uniquely determined by $\operatorname{tb}(K)$ and $\operatorname{rot}(K)$ as follows:

(1) If $\operatorname{rot}(K) \neq 0$ (Figure 12(a)), then

$$
\begin{aligned}
& \operatorname{tb}(K)=-(2 t+1+s), \\
& \operatorname{rot}(K)= \begin{cases}s & \text { if the leftmost cusp is a down cusp, } \\
-s & \text { if the leftmost cusp is an up cusp. }\end{cases}
\end{aligned}
$$

(2) If $\operatorname{rot}(K)=0$ (Figure 12(b)), then

$$
\operatorname{tb}(K)=-(2 t+1) .
$$

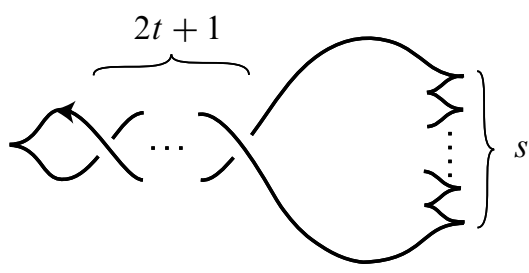

(a)

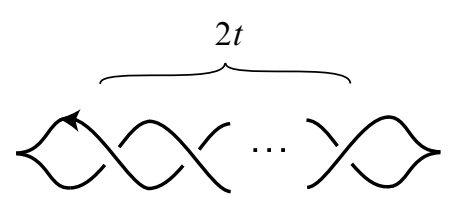

(b)

Figure 12. Legendrian unknot in standard form: (a) $\operatorname{rot}(K)>0$ (reverse orientation gives $\operatorname{rot}(K)<0$ ) (b) $\operatorname{rot}(K)=0$.

We define the standard form embeddings for Legendrian graphs below.

Definition 5.5 (1) A planar Legendrian realization of the lollipop graph is in standard form if it is the lift of a front projection consisting of one front projection of an unknot $U$ in standard form as in Figure 12 and a nonstabilized arc at the lower right cusp of the unknot. The arc can sit in one of two ways with respect to the other edge segments coming together at the vertex. The planar Legendrian realization of the lollipop graph is in standard form $A$ or $B$ if the cut edge sits as in Figure 13(a) or (b), respectively.

(2) A planar Legendrian realization of the handcuff graph is in standard form if it is the lift of a front projection consisting of two noncrossing front projections of unknots $U_{1}$ and $U_{2}$ each in standard form as in Figure 12, one on the left and one on the right, and a nonstabilized arc between the lower right cusp of the unknot on the left and the 


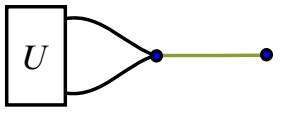

(a)

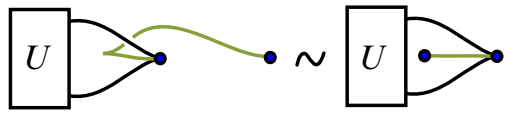

(b)

Figure 13. Planar Legendrian realization of the lollipop graph in (a) standard form $A$, (b) standard form $B$

leftmost cusp of the unknot on the right. The arc can sit in one of two ways with respect to the other edge segments coming together at each vertex. The planar Legendrian realization of the handcuff graph is in standard form $A A, A B, B A$ or $B B$ if the cut edge sits as in Figure 14(a), (b), (c) or (d), respectively.

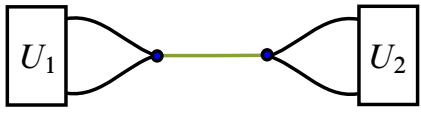

(a)

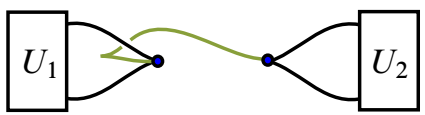

(c)

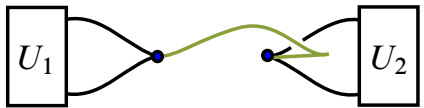

(b)

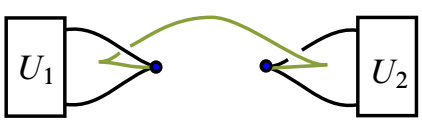

(d)

Figure 14. Planar Legendrian realization of the handcuff graph in (a) standard form $A A$, (b) standard form $A B$, (c) standard form $B A$, (d) standard form $B B$

Figure 15 shows an example of a handcuff graph in standard form $A A$ with both unknotted components with rot $\neq 0$.

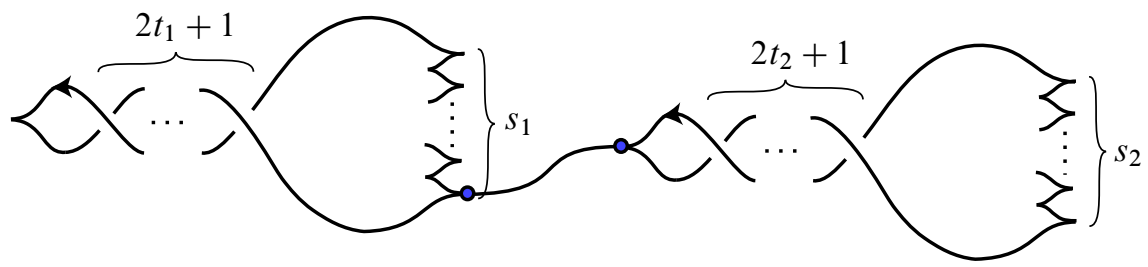

Figure 15. Legendrian handcuff graph in standard form $A A$ with both unknotted components with rot $>0$

Lemma 5.6 Let $G$ be a Legendrian graph consisting of a Legendrian knot and a cut edge connected to it. Through Legendrian isotopy the cut edge can be moved to be connected at any point of the knot. 
Proof We work with a front projection of the Legendrian graph $G$. Away from the cusps the cut edge can be moved by planar isotopy. A cut edge can be passed through a right cusp as in Figure 16 (below or above, depending on how it sits with respect to the cusp). Passing from the lower strand to the upper strand of a right cusp can be obtained by vertical reflection of the two illustrated cases. Diagrams for passing through a left cusp can obtained by horizontal reflection of the diagrams for the right cusp.
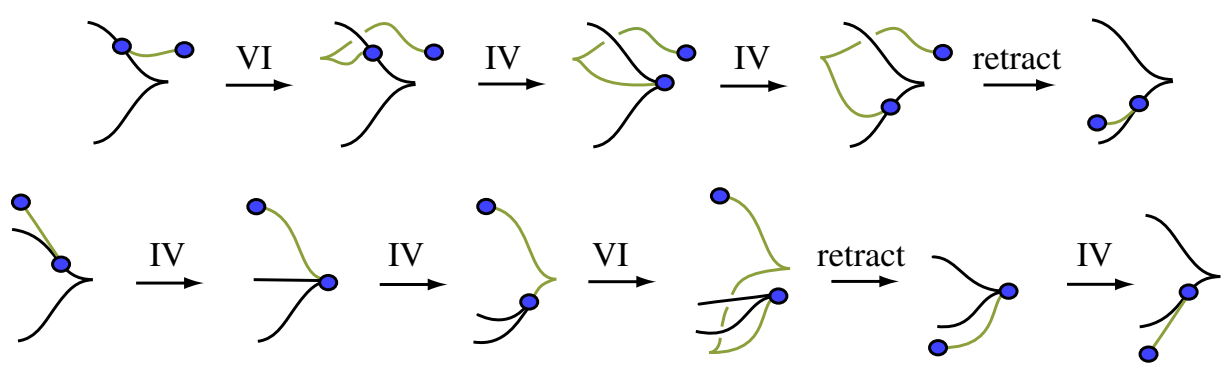

Figure 16. Sliding the cut edge past a right cusp

Theorem 5.7 A pair (tb, rot) determines exactly two Legendrian isotopy classes for a planar Legendrian realization of the lollipop graph.

Proof We construct a Legendrian isotopy between $L$ and one of the two standard forms. We fix the notation: $v_{1}$ is the valence three vertex of $L, v_{2}$ is the valence one vertex of $L, U$ is the loop edge of $L$ and $e$ is the cut edge of $L$. We work with a front projection of $L$.

Step 1 (Remove the crossings of the cut edge with itself and with $U$.) Starting from $v_{2}$ towards $v_{1}$, retract the edge $e$ in a neighborhood of $v_{1}$ and remove all its self crossings in the front projection and all crossings between $e$ and $U$.

Step 2 (Put $U$ in standard form.) Change $L$ by Legendrian isotopy in a neighborhood of $v_{1}$ such that the unknot $U$ is everywhere smooth. Eliashberg and Fraser [5] proved the existence a unique unknot in standard form which is Legendrian isotopic to $U$. Take $U$ to standard form through Legendrian isotopy while keeping $v_{2}$ and its neighborhood containing $e$ away from the isotopy. We can do this by sliding the cut edge when necessary, as in Lemma 5.6.

Step 3 (Slide the cut edge to the lower right cusp of $U$.) Using Lemma 5.6, slide the cut edge so that it connects to the rest of the graph at the lower right cusp of $U$. Starting from $v_{2}$ towards $v_{1}$, retract the edge $e$ in a neighborhood of $v_{1}$ and remove all self crossings in the front projection and all crossings between $e$ and $U$. 
Now the graph is in one of the two standard forms. Since a standard form of the unknot is uniquely determined by tb and rot, each of the standard forms of the lollipop graph are also determined by tb and rot. Thus, we have two Legendrian isotopy classes for the lollipop graph for each pair (tb, rot).

Theorem 5.8 A pair (tb, rot) determines exactly four Legendrian isotopy classes for a planar Legendrian realization of the handcuff graph.

Proof We construct a Legendrian isotopy between $L$ and one of the four standard forms. We fix the notation: $v_{1}$ and $v_{2}$ are the two vertices of $L, U_{1}$ and $U_{2}$ are the two loop edges of $L$ and $e$ is the cut edge of $L$. We work with a front projection of $L$.

Step 1 (Make $U_{1}$ and $U_{2}$ disjoint in the front projection.) Since the graph $L$ is topologically equivalent to the embedding in Figure 11(b), the two unknots $U_{1}$ and $U_{2}$ bound disks $D_{1}$ and $D_{2}$ which are disjoint from each other and disjoint from the rest of the graph. Shrink the disks $D_{1}$ and $D_{2}$ in small enough neighborhoods of $v_{1}$ and $v_{2}$ such that there exists no crossing between $U_{1}$ and $U_{2}$ in the front projection.

Step 2 (Remove crossings of the cut edge with itself and with $U_{1}$ and $U_{2}$.) There exists an embedded 2-sphere $S_{1}$ such that $U_{1}$ is contained in the 3-ball $B_{1}$ bounded
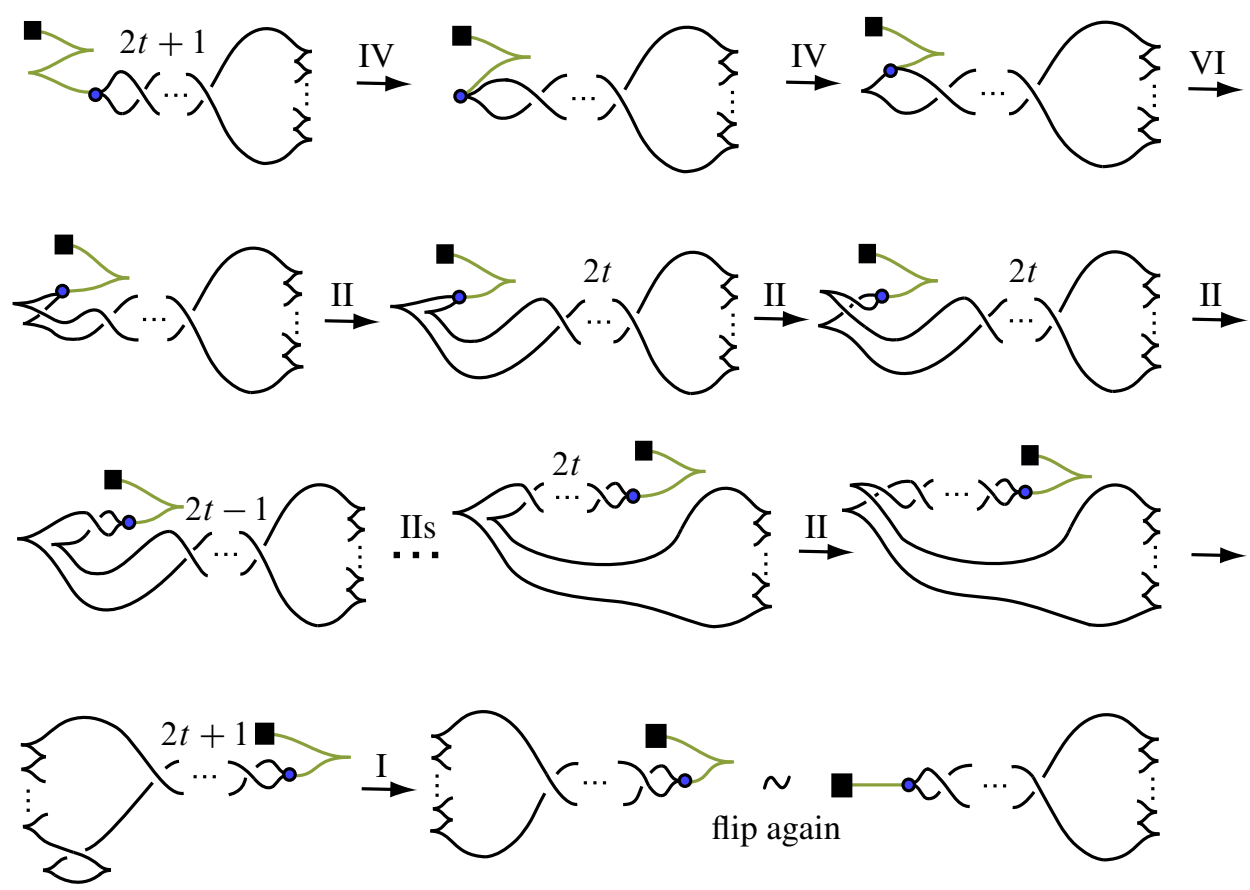

Figure 17. Flip of $U_{2}$ undoes the stabilization in the cut edge for the case when the cut edge connects outside the cusp at $v_{2}$. 
by $S_{1}$ and $S_{1}$ intersects the cut edge at one point, $w_{1}$. Shrink $B_{1}$ in a small neighborhood of $w_{1}$. Starting from $w_{1}$, retract the cut edge while carrying along the neighborhood of $w_{1}$ and undo its knotting outside of $B_{1}$ as well as all crossings with $U_{2}$.

There exists an embedded 2-sphere $S_{2}$ disjoint from the sphere $S_{1}$ such that $U_{2}$ lies in the 3-ball $B_{2}$ bounded by $S_{2}$ and $S_{2}$ intersects the cut edge at one point, $w_{2}$. Shrink $B_{2}$ in a small neighborhood of $w_{2}$. This move may introduce a crossing between $e$ and $U_{2}$ in the front projection. Starting from $w_{2}$, retract the cut edge while carrying along the neighborhood of $w_{2}$ and undo its knotting outside of $B_{2}$ as well as all crossings between the cut edge and $U_{1}$ in the front projection.

Step 3 (Put $U_{1}$ and $U_{2}$ in standard form, slide one end of the cut edge to the lower right cusp of $U_{1}$ and slide the other end to the left cusp of $U_{2}$.) Take $U_{1}$ into a small neighborhood of $v_{1}$. Modify $U_{2}$ through a Legendrian isotopy which takes it to the unknot in standard form having the assigned tb and rot. By sliding the cut edge repeatedly as in Lemma 5.6 we can keep $v_{1}$ and $U_{1}$ away for this isotopy. Once $U_{2}$ is in standard form, using Lemma 5.6, slide the cut edge so that it connects to $U_{2}$ at the left cusp of $U_{2}$. The cut edge can sit in two ways with respect to the other two edge segments at this cusp.

Leaving $U_{2}$ in standard form and leaving $e$ connected to $U_{2}$ at the left cusp, move $U_{1}$ and $e$ through Legendrian isotopy so that the front projection of $U_{1}$ lies outside and to the left of the bounded region in the plane determined by the front projection of $U_{2}$.

Modify $U_{1}$ through a Legendrian isotopy which takes it to the unknot in standard form having the assigned tb and rot. By sliding the cut edge repeatedly as in Lemma 5.6 without contracting it we leave $U_{2}$ unchanged. Once $U_{1}$ is in standard form, using Lemma 5.6 slide the cut edge without contracting it so that it connects to $U_{1}$ at the lower right cusp of $U_{1}$.

Step 4 (Undo stabilizations of the cut edge and reach one of the standard forms.) The cut edge can connect in two ways at $v_{2}$ relative to the other two edge segments: outside the cusp or inside the cusp.

(1) If the cut edge connects outside the cusp, then the stabilizations of the cut edge can be removed by flipping $U_{2}$ horizontally, as in Figure 17. The other type of stabilization is solved by reflecting the diagrams. After undoing the additional stabilizations the graph is in one of the standard forms $A A$ or $B A$, depending on how the cut edge sits at $v_{1}$ relative to the other two edge segments. 
(2) If the cut edge connects inside the cusp, then the stabilizations of the cut edge can be removed by flipping $U_{2}$ horizontally, as in Figure 18. The other type of stabilization is solved by reflecting the diagrams. After undoing the additional stabilizations the graph is in one of the standard forms $A B$ or $B B$, depending on how the cut edge sits at $v_{1}$ relative to the other two edge segments.
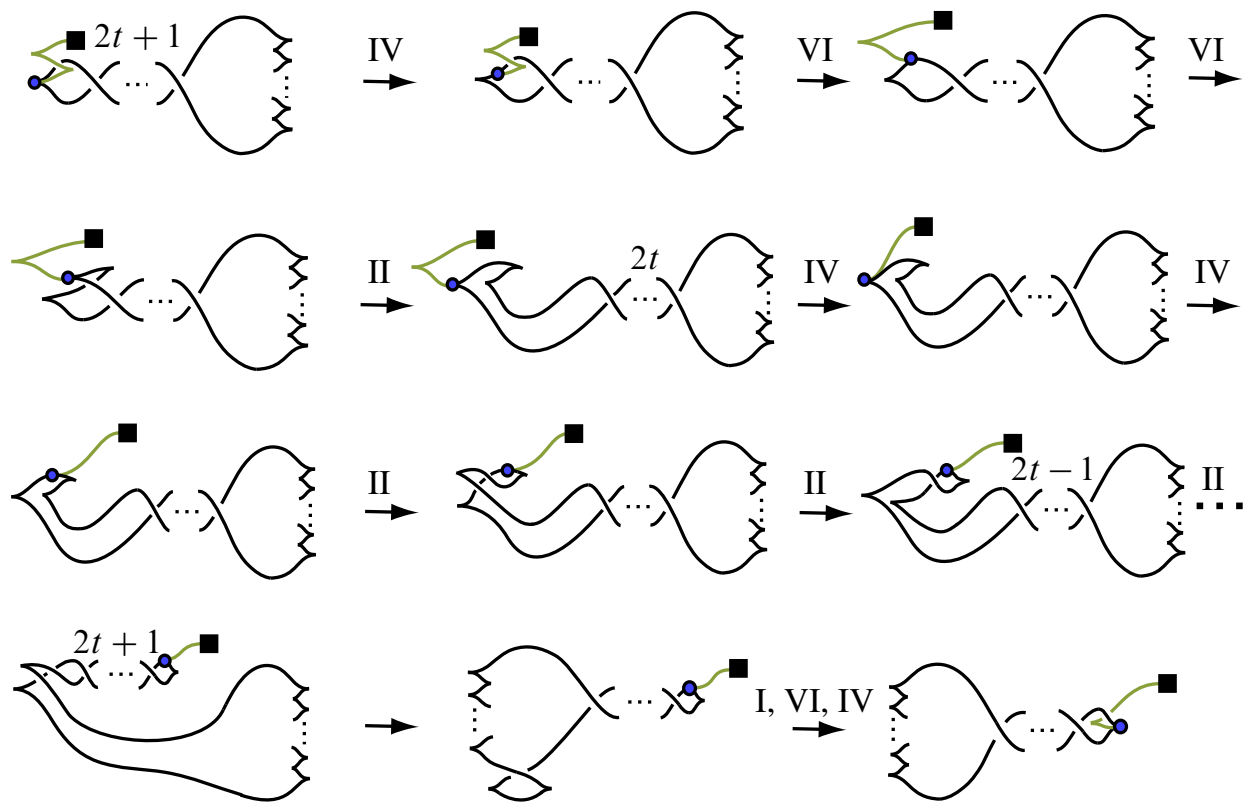

Figure 18. Flip of $U_{2}$ undoes the stabilization in the cut edge for the case when the cut edge connects inside the cusp at $v_{2}$.

Remark 5.9 If we replace the two unknotted cycles by cycles which are knots whose Legendrian type is determined by tb and rot, the theorem still holds.

\section{References}

[1] S Baader, M Ishikawa, Legendrian graphs and quasipositive diagrams, Ann. Fac. Sci. Toulouse Math. 18 (2009) 285-305 MR2562830

[2] J H Conway, C M Gordon, Knots and links in spatial graphs, J. Graph Theory 7 (1983) 445-453 MR722061

[3] F Ding, H Geiges, Legendrian knots and links classified by classical invariants, Commun. Contemp. Math. 9 (2007) 135-162 MR2313510

[4] Y Eliashberg, Contact 3-manifolds twenty years since J Martinet's work, Ann. Inst. Fourier (Grenoble) 42 (1992) 165-192 MR1162559 
[5] Y Eliashberg, M Fraser, Topologically trivial Legendrian knots, J. Symplectic Geom. 7 (2009) 77-127 MR2496415

[6] J B Etnyre, K Honda, Knots and contact geometry I: Torus knots and the figure eight knot, J. Symplectic Geom. 1 (2001) 63-120 MR1959579

[7] H Geiges, An introduction to contact topology, Cambridge Studies in Adv. Math. 109, Cambridge Univ. Press (2008) MR2397738

[8] E Giroux, Géométrie de contact: de la dimension trois vers les dimensions supérieures, from: "Proceedings of the International Congress of Mathematicians, Vol. II (Beijing, 2002)", (T Li, editor), Higher Ed. Press, Beijing (2002) 405-414 MR1957051

[9] L H Kauffman, Invariants of graphs in three-space, Trans. Amer. Math. Soc. 311 (1989) 697-710 MR946218

[10] C Kuratowski, Sur le probléme des courbes gauches en topologie, Fund. Math. 15 (1930) 271-283

[11] K Mohnke, Legendrian links of topological unknots, from: "Topology, geometry, and algebra: interactions and new directions (Stanford, CA, 1999)”, (A Adem, G Carlsson, R Cohen, editors), Contemp. Math. 279, Amer. Math. Soc. (2001) 209-211 MR1850749

[12] N Robertson, P D Seymour, R Thomas, Linkless embeddings of graphs in 3-space, Bull. Amer. Math. Soc. 28 (1993) 84-89 MR1164063

[13] H Sachs, On spatial representations of finite graphs, from: "Finite and infinite sets, Vol. I, II (Eger, 1981)”, (A Hajnal, L Lovász, V T Sós, editors), Colloq. Math. Soc. János Bolyai 37, North-Holland, Amsterdam (1984) 649-662 MR818267

Department of Mathematics and Statistics, Smith College

44 College Lane, Northampton MA 01060, USA

Department of Mathematics, Occidental College

1600 Campus Road, Los Angeles CA 90041-3314, USA

dodonnol@smith.edu, pavelescu@oxy.edu

Received: 11 October 2011 Revised: 31 January 2012 